\title{
A "real life" investigation on the prescriptive habits among Italian andrologists: The "CONSER" survey from Italian Society of Andrology (SIA) on Sildenafil oral film
}

\author{
Alessandro Palmieri ${ }^{1}$, Mauro Silvani ${ }^{2}$, Bruno Giammusso ${ }^{3}$, Giovanni Liguori ${ }^{4}$, Nicola Mondaini ${ }^{5}$, \\ Fabrizio Palumbo ${ }^{6}$, Stefano Pecoraro ${ }^{7}$, Oreste Risi ${ }^{8}$, Salvatore Sansalone ${ }^{9}$, Fabrizio Ildefonso Scroppo ${ }^{10}$, \\ Alessandro Zucchi ${ }^{11}$, Paolo Verze ${ }^{1}$, Marco Capece ${ }^{1}$, Tommaso Cai ${ }^{12}$ \\ ${ }^{1}$ Department of Urology, University of Naples, Naples, Italy; ${ }^{2}$ Urology Unit, Hospital "degli Infermi", Biella, Italy; \\ ${ }^{3}$ Morgagni Clinic, Catania, Italy; ${ }^{4}$ Department of Urology, University of Trieste, Trieste, Italy; ${ }^{5}$ Urology Unit, Nuovo San Giovanni \\ di Dio Hospital, Florence, Italy; ${ }^{6}$ Urology Unit, San Giacomo Hospital, Monopoli, Bari, Italy; ${ }^{7}$ Malzoni Center, Avellino, Italy; \\ ${ }^{8}$ Neuro-Urology Unit, Treviglio, Bergamo, Italy; ${ }^{9}$ Department of Urology, University of Tor Vergata, Rome, Italy; \\ ${ }^{10}$ Urology Unit, Ospedale di Circolo di Varese, Varese, Italy; ${ }^{11}$ Department of Urology, University of Perugia, Perugia, Italy. \\ 12 Department of Urology, Santa Chiara Hospital, Trento, Italy.
}

\begin{abstract}
Summary Even if oral type 5 phosphodiesterase inhibitors (PDE5i) seem an effective treatment for erectile dysfunction (ED), the drop-out is high among patients. For this reason, pharmaceutical companies are encouraged to develop new administration routes, such as the orally disintegrating film. The aim of this study was to analyse the prescription habit of Italian andrologists affiliated to Italian Society of Andrology (SIA) in the era of new oro-dispersible formulation of sildenafil. A 12-items dedicated questionnaire has been distributed to 77 urologists andrologists. As a result of the questionnaire, sildenafil is still the preferred drug of Italian andrologists as it is considered the safest and the most effective. It combines the speed of action and the discretion of the intake that are very important issues for the adherence to the treatment according to the Italian sample. Physicians have also reported the positive feedback of the patients taking sildenafil film as they consider the oro-dispersible formulation either comparable or superior to the old tablet. In conclusion this new formulation has given a new life to an old molecule like sildenafil, and Italian andrologists considered this new pharmaceutical formulation as a good tool to improve the patient's adherence to the treatment and quality of life.
\end{abstract}

KEY WORDS: Erectile dysfunction; Sildenafil; Oro-dispersible; Quality of life; Survey; Andrology.

Submitted 6 May 2019; Accepted 10 May 2019

\begin{abstract}
INTRODUCTION
Over the last 20 years the use of phosphodiesterase type 5 inhibitors (PDE5i) has revolutionized classification and treatment of erectile dysfunction (ED) becoming a firstline therapy as recommended by the guidelines of all major scientific societies (1-3).

After the marketing of Viagra in 1998, the various international drug companies have approved the use of different molecules characterized by identical mechanisms of action but with different pharmacokinetic properties: in chronological order Tadalafil, Vardenafil and Avanafil. Although international medical literature is consistent in
\end{abstract}

defining PDE5i efficacy in over $80 \%$ of cases of ED, the fact remains that only a minority of men with ED currently seek help by consulting an Andrologist and 60$70 \%$ of those who decide to undertake specific medical care stop treatment for a variety of reasons (4-6).

So-called "adherence" to therapy is therefore a primary determinant in the success of treatment but unfortunately there are many factors that often cause a drop-out from the treatment: specialists who prescribe PDE5is and give inadequate information to the patient often as result of a superficial andrological visit, expectations of a patients who take ineffective drugs, side effects and high costs. Despite the enormous amount of data available in the literature, identification of the specific reasons for patients suspending ED pharmacological treatment is extremely difficult, above all because "evidence-based" studies are lacking. So far, no randomized clinical trial (RCT) has been published which compares the efficacy and tolerability of Sildenafil, Vardenafil, Tadalafil and Avanafil.

The reasons for this gap are largely attributable to the different characteristics of pharmacokinetics, bioavailability and methods of administration of these drugs which affect absorption time, duration and onset of action, methods of use, etc., making comparisons impossible. In addition, published subjective assessment studies, based predominantly on patient opinion, have serious design defects such as comparisons between different drug assays and/or short treatment duration (7-11). Despite these difficulties some authors have attempted, for the very first time, a meta-analysis "Trade off" aimed at identifying what could be the optimal PdE5i in the treatment of ED relative to efficacy and incidence of side effects (5). This analysis was performed on 82 studies (47.626 patients) for efficacy evaluations and 72 studies (20.325 patients) on different molecules' adverse events. Analysis of available data allowed for the identification of Sildenafil $50 \mathrm{mg}$ as the most effective drug but was burdened by a higher incidence of adverse events compared to competitors. Authors conclude that Sildenafil, first put on the mar-

No conflict of interest declared. 
ket more than 20 years ago, at a dosage of $50 \mathrm{mg}$ can be considered an initial choice in the treatment of ED of patients for whom high drug efficacy is a priority.

Although Sildenafil is the oldest molecule available on the market, it is the first one engineered in oro-dispersible tablets and oro-dispersible film to improve the pharmacokinetics characteristics with the latter being the latest (12). Starting from these considerations it is crucial to understand the efficacy of the drugs in relation to the various formulations and how these ones impact on the specialists' clinical practice.

The aim of this work is to add new elements to define how the introduction of a new sildenafil oro-soluble film has changed ED management in a selected group of Italian andrologists from Italian Society of Andrology (SIA).

\section{Survey}

In the period between May 2017 and December 2017, the Italian Society of Andrology (SIA) conducted a survey on PDE5i involving numerous Italian Andrologists. The project is named "CONSER" ("conservare l'erezione").

A 12-items ad-hoc created and dedicated questionnaire has been sent via registered mail to 77 andrologists affiliated to SIA and homogenously distributed on the wide Italian regions.

Some remarkable statistical data have been extrapolated from the overall analysis of the answers, which envisages the prescription reality of PDE5i, with attention given, not only from the point of view of the andrological specialist, but also regarding the needs of the patient which, if disregarded, are the primary cause of poor therapeutic compliance. The first interesting data resulting from analysis of the survey, is that according to the andrologists only $9 \%$ of the patients have been completely satisfied with the pharmacological therapy they had been prescribed. The problems encountered were mainly cost/reimbursement and low efficacy, thus these factors are the major issues involved in either the specialist prescription and in patient's adherence to the therapy (Tables 1, 2).

According to the survey, the most important features a PDE5i should ensure are the speed of action and the duration of the effect for either the specialist and the patient (Figures 1,2).

Regarding to the pharmacokinetics, it is commonly believed that the starting dosage depends on the type of PDE5i used. Almost two thirds of the patients preferred Sildenafil as first therapeutic choice (64.3\%), followed by Tadalafil, Vardenafil e Avanafil (20.3\%, 8.5\% and $6.8 \%$ respectively). Sildenafil is still the preferred drug of Italian andrologists as it is considered the safest and the most effective. Finally, with regard to the new oro-soluble film formulation of Sildenafil, physicians believe that the new administration route combines the advantages of the speed of action and the discretion of the intake. Furthermore, it has been reported that patients easily accept this
Table 1.

Which of these aspects can represent a limit to your full satisfaction in the therapeutic approach to the patient with erectile deficit?

\begin{tabular}{|l|c|c|}
\hline Answer & Number & $\%$ \\
\hline Cost/refund & 49 & 63.6 \\
\hline Dosage & 5 & 6.5 \\
\hline Side effects & 7 & 9.1 \\
\hline Poor efficacy & 16 & 20.8 \\
\hline Total & $\mathbf{7 7}$ & $\mathbf{1 0 0}$ \\
\hline
\end{tabular}

Table 2.

Which of these aspects related to therapy represent a limit to the patient?

\begin{tabular}{|l|c|c|}
\hline Answer & Number & $\%$ \\
\hline Cost/refund & 53 & 68.8 \\
\hline Dosage & 1 & 1.3 \\
\hline Side effects & 12 & 15.6 \\
\hline Poor efficacy & 11 & 14.3 \\
\hline Total & $\mathbf{7 7}$ & $\mathbf{1 0 0}$ \\
\hline
\end{tabular}

Figure 1.

What are the pharmacological characteristics of a PDE5-i that, according to your clinical practice, are decisive for therapeutic success?

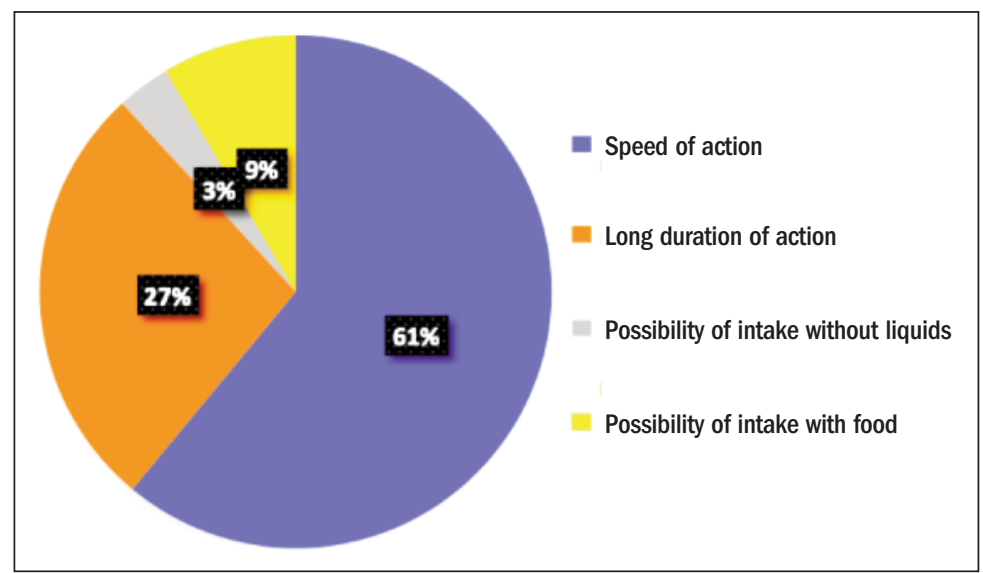

Figure 2.

What do patients ask from a PDE 5-i?

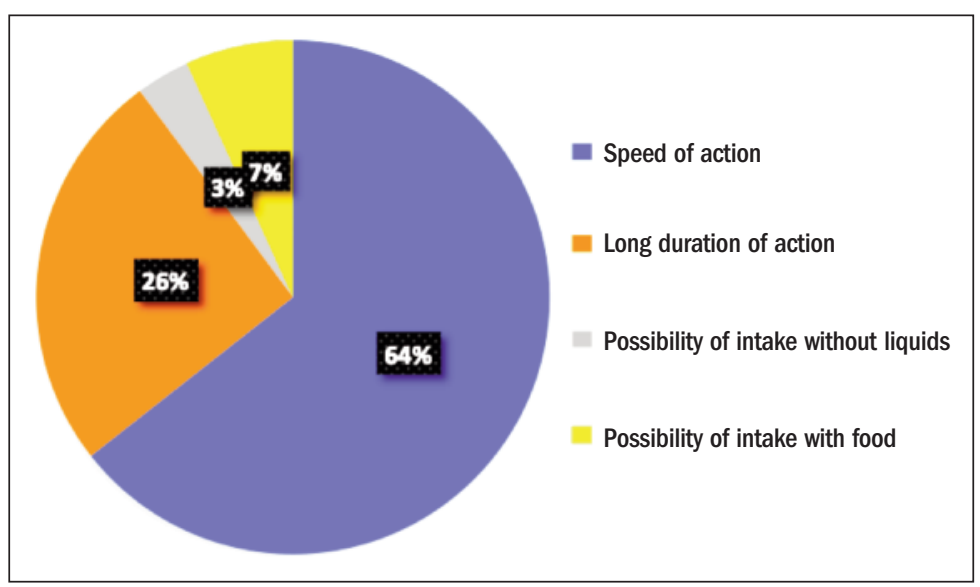


Table 3.

How is the oral formulation received by patients?

\begin{tabular}{|l|c|c|}
\hline Answer & Number & $\%$ \\
\hline Optimally & 21 & 35.6 \\
\hline Very well & 36 & 61 \\
\hline $\begin{array}{l}\text { Without difference compared } \\
\text { to the other formulations }\end{array}$ & 2 & 3.4 \\
\hline With mistrust & 0 & 0 \\
\hline Total & $\mathbf{5 9}$ & $\mathbf{1 0 0}$ \\
\hline
\end{tabular}

form of treatment because of its efficacy, that is either comparable or superior to other oral formulations (Table 3). The new formulation of oro-dispersible Sildenafil film was prescribed by most of the andrologists involved in the survey with extreme confidence both in naïve patients and in the presence of co-morbidities.

\section{Discussion}

In the recent years alternative drug-delivery systems have been developed to improve patient compliance and adherence to the therapy. With regards to the drug absorption, the route of administration is the main factor, and the variables influencing it are represented by the pharmaceutical formulation (coefficient of distribution /dissolubility) and the characteristics of the absorbing surface (extension, permeability, vascularization). Having stated that, the best results can be obtained with a greater contact surface between the drug and the mucous membranes which are highly vascularized, a good distribution coefficient of the drug (liposolubility/hydrosolubility ratio) and good permeability of the contact surface (e.g. oral mucous membrane).

Previous studies have evaluated the efficacy of Sildenafil and tested its absorption in the oral mucosa. A first study by El-Rashidy et al. (13) proposed a sublingual (delitescent) formulation for Sildenafil. The formulation involved the use of the drug (Sildenafil), an osmotic agent (mannitol), a hydrophilic carrier (microcrystalline cellulose), and a water-soluble polymer (cellulose derivative). These additional components to the drug had the task of facilitating the transmucosal passage of Sildenafil by favouring its absorption (carrier). Unfortunately there are no data available on the results. A second study proposed by De Siati in 2003 (14) conducted in Italy proposed an evaluation of the effects of oral intake of Sildenafil (the "old" tablets formulation) for 3 months by comparing the data after a further 3 months of therapy in which the patient pulverized the tablet and placed the powder produced under the tongue. The results obtained showed that the time for the drug to be effective was halved: $62.8 \mathrm{~min}$. (whole tablets) vs. 29.3 min. (sublingual). In the same study all patients stated that they preferred the sublingual route for the most rapidity of action of the drug. Wang in 2008 (15) pointed out that Sildenafil is a lipophilic molecule and therefore can easily be absorbed at the sublingual level however noted that poor solubility in saliva is a limiting factor for absorption at the level of the oral mucosa and this parameter therefore needs to be improved (16).

After about 10 years, research and technology have suc- ceeded in developing new formulations, as in the case of Sildenafil in delitescent leaflets (SILER) which can be absorbed at the level of the oral mucosa. The new formulation in orodispersible film now has all the characteristics to facilitate sublingual absorption as it is composed of Sildenafil, a lipophilic molecule easily absorbed by the oral mucosa, maltodestrine which acts as a "filmogenic" and a solubilizing agent. The maltodestrine also works as a carrier that facilitates its absorption through the oral mucosa $(17,18)$; stimulating salivation agents such as citric acid, which favor its rapid disintegration, modifying the salivary $\mathrm{pH}$ towards acidity and in this way improving the absorption of the drug $(19,20)$.

In a recent paper the "old" oral tablets have been compared to the new sildenafil film whose median action time was 20 minutes. The greater rapidity of absorption, attributed to its pre-gastric absorption, ratified its superiority to the traditional tablet (21).

Despite its twenty years-long history, our survey declares Sildenafil the first choice of Italian andrologists. Our analysis cannot estimate whether these results are related to the marketing of the new formulations or to the patients' feedback, however both hypothesis are not mutually exclusive. Therefore it is likely that both have contributed to the actual Italian state of affairs.

The analysis also demonstrate that the new formulation of Sildenafil is well known to Italian andrologists and that they think the patients would prefer the characteristics of the new formulation to the old one. The new administration route, developed by IBSA (Lugano, Switzerland) and approved in Europe at doses of 25, 50, 75, and $100 \mathrm{mg}$, is considered a safe and effective alternative to the conventional tablets. Finally it gives the possibility of chosing a 75 mg dosage that previous formulations did not consider.

\section{CONCLUSIONS}

In conclusion, in the world of PDE5 inhibitors orodispersible film of Sildenafil represents a real technological innovation with a product that is easy to ingest, with great rapidity of action and fewer side effects. In particular, we demonstrated that the advantage of the new formulation of Sildenafil in oro-dispersible film is represented, not only by the fact that it can be taken discreetly and without water, but above all by the possibility that the drug can be absorbed, at least in part, directly at the level of the oral mucosa, entering immediately into circulation with no first liver passage.

\section{REFERENCES}

1. Hatzimouratidis K. Can we cure erectile dysfunction? Eur Urol. 2010; 58:249-50.

2. Eardley I, Donatucci C, Corbin J, et al. Pharmacotherapy for erectile dysfunction. J Sex Med. 2010; 7:524-40.

3. Porst H, Burnett A, Brock G, et al. SOP conservative (medical and mechanical) treatment of erectile dysfunction. J Sex Med. 2013; 10:130-71.

4. Corona G, Mondaini N, Ungar A, et al. Phosphodiesterase type 5 (PDE5) inhibitors in erectile dysfunction: the proper drug for the proper patient. J Sex Med. 2011; 8:3418-32. 
5. Chen L, Staubli SE, Schneider MP, et al. Phosphodiesterase 5 inhibitors for the treatment of erectile dysfunction: a trade-off network meta-analysis. Eur Urol. 2015; 68:674-80.

6. Fagelman E, Fagelman A, Shabsigh R. Efficacy, safety, and use of sildenafil in urologic practice. Urology 2001; 57:1141-4.

7. Govier F, Potempa AJ, Kaufman J, et al. A multicenter, randomized, double-blind, crossover study of patient preference for tadalafil $20 \mathrm{mg}$ or sildenafil citrate $50 \mathrm{mg}$ during initiation of treatment for erectile dysfunction. Clin Ther. 2003; 25:2709-23.

8. von Keitz A, Rajfer J, Segal S, et al. A multicenter, randomized, double-blind, crossover study to evaluate patient preference between tadalafil and sildenafil Eur Urol. 2004; 45:499-507.

9. Ströberg P, Murphy A, Costigan T. Switching patients with erectile dysfunction from sildenafil citrate to tadalafil: results of a European multicenter, open-label study of patient preference. Clin Ther. 2003; 25:2724-37.

10. Althof SE. Quality of life and erectile dysfunction. Urology. 2002; 59:803-10.

11. Maurice DH, Wilson LS, Rampersad SN, et al. Cyclic nucleotide phosphodiesterases (PDEs): coincidence detectors acting to spatially and temporally integrate cyclic nucleotide and non-cyclic nucleotide signals., Biochem Soc Trans. 2014; 42:250-6.

12. De Toni L, De Rocco Ponce M, Franceschinis E, et al. Sublingual administration of sildenafil oro-dispersible film: new profiles of drug tolerability and pharmacokinetics for PDE5 inhibitors. Front Pharmacol. 2018; 6; 9:59.

13. El-Rashidy R. Controlled Release of Sildenafil Delivered by Sublingual or Buccal Administration. WO 00/5477. 2002.
14. De Siati M, Saugo M, Franzolin N. The start of pharmacological activity after sublingual administration of sildenafil citrate in 30 patients affected by erectile dysfunction. Arch Ital Urol Androl. 2003; 75:18-20.

15. Wang Y, Chow MSS, Zuo Z. Mechanistic analysis of $\mathrm{pH}$-dependent solubility and trans-membrane permeability of amphoteric compounds: Application to sildenafil International Journal of Pharmaceutics. 2008; 352:217-224

16. Rathbone MJ, Ponchel G, Ghazali FA. Systemic oral mucosal drug delivery and delivery systems. In: Rathbone, M.J. (Ed.), Oral Mucosal Drug Delivery. Marcel Dekker, Inc., New York, 2004.

17. Smyth HDC, Hickey AJ. Carriers in drug powder delivery. American Journal of Drug Delivery. 2005; 3:117-132.

18. Parikh A, Agarwal S, Raut K. A Review on applications of Maltodextrin in pharmaceutical industry. IJPBS 2014; 4:67-74.

19. Shweta Kalyan, Mayank Bansal. Recent Trends in the Development of Oral dissolving Film. International Journal of Pharm Tech Research. 2012; 4:725-733.

20. Kathpalia H, Gupte A. An Introduction to fast dissolving oral thin film drug delivery systems: a review. Current Drug delivery. 2013; 10:667-684.

21. Cocci A, Capece M, Cito G, et al. Effectiveness and safety of orodispersible sildenafil in a new film formulation for the treatment of erectile dysfunction: comparison between sildenafil 100-mg filmcoated tablet and 75-mg oro-dispersible film. J Sex Med. 2017; 14:1606-1611.

\section{Correspondence}

Alessandro Palmieri, MD

Paolo Verze, MD

Marco Capece, MD

Department of Urology, University of Naples, Naples (Italy)

Mauro Silvani, MD

Urology Unit, Hospital "degli Infermi", Biella (Italy)

Bruno Giammusso, MD

Morgagni Clinic, Catania (Italy)

Giovanni Liguori, MD

Department of Urology, University of Trieste, Trieste (Italy)

Nicola Mondaini, MD

Urology Unit, Nuovo San Giovanni di Dio Hospital, Florence (Italy)

Fabrizio Palumbo, MD

Urology Unit, San Giacomo Hospital, Monopoli, Bari (Italy)

Stefano Pecoraro, MD

Malzoni Center, Avellino (Italy)

Oreste Risi, MD

Neuro-Urology Unit, Treviglio, Bergamo (Italy)

Salvatore Sansalone, MD

Department of Urology, University of Tor Vergata, Rome (Italy)

Fabrizio Ildefonso Scroppo, MD

Urology Unit, Ospedale di Circolo di Varese, Varese (Italy)

Alessandro Zucchi, MD

Department of Urology, University of Perugia, Perugia (Italy)

Tommaso Cai, MD

ktommy@libero.it

Department of Urology, Santa Chiara Hospital - Largo Medaglie d'Oro 9, Trento (Italy) 